\title{
Masses of asteroids and total mass of the main asteroid belt
}

\author{
E. V. Pitjeva ${ }^{1}$ and N. P. Pitjev ${ }^{2}$ \\ ${ }^{1}$ Institute of Applied Astronomy, Russian Academy of Sciences, \\ Kutuzov Quay 10, 191187 St.Petersburg, Russia \\ email: evp@ipa.nw.ru \\ ${ }^{2}$ St. Petersburg State University, \\ Universitetski pr. 28, 198504, Russia \\ email: ai@astro.spbu.ru
}

\begin{abstract}
An estimation of the mass of the main asteroid belt was made on the basis of the new version of EPM2014 ephemerides of the Institute of Applied Astronomy of Russian Academy of Sciences using about 800000 positional observations of planets and spacecraft. We obtained the individual estimations of masses of large asteroids from radar data, as well as estimates of the masses of asteroids by using known diameters and estimated average densities for the three taxonomic types $(\mathrm{C}, \mathrm{S}, \mathrm{M})$, and used the known mass values of binary asteroids and asteroids to which spacecraft approached. A two-dimensional homogeneous annulus with dimensions corresponding observed width of the main asteroid belt (2.06 au and $3.27 \mathrm{au}$ ) was used instead of a previous massive one-dimensional ring for modeling total perturbations from small asteroids. The obtained value of the total mass of the main asteroid belt is $(12.25 \pm$ $0.19) 10^{-10} M_{\odot}$.
\end{abstract}

Keywords. methods: analytical, data analysis, numerical; ephemerides; minor planets, asteroids

\section{Introduction}

Perturbations from asteroids significantly affect the orbits of planets and should be taken into account when high-accuracy planetary ephemerides are constructed. On the other hand, from an analysis of motion of the major planets, some physical parameters of the asteroids may be obtained. To improve the estimation of the mass of the main asteroid belt and its gravitational influence on the motion of bodies in the solar system, the updated database of asteroids and observations of planets and spacecraft is used, as well as the new version of the EPM2014 planetary ephemerides. The infrared data indicate that the main belt zone may contains millions of asteroids with a diameter of $1 \mathrm{~km}$ or more (Tedesco \& Desert 2002, Tedesco et al. 2005). Moreover, fragmentation of asteroids happens during their collisions (Petit et al. 2001). Therefore, the number of small bodies in the asteroid belt must be greater by orders of magnitude than the given estimate. In addition, there is also a dust component.

Asteroids with orbits located between the resonances of 4:1 and 2:1 of the mean motion with Jupiter compose the majority of the main belt bodies. This area of the belt (the radial distance between 2.06 and $3.27 \mathrm{au}$ ) contains more than $90 \%$ of all numbered asteroids, and these distances can be considered, respectively, as the inner and outer boundaries of the main belt; outside of it, the number of asteroids decreases sharply.

The number of position observations used has increased by an order since EPM2000 (2001) and amounts for EPM2014 more than 800000 data of different types (Table 1), most of them are the new high-accuracy radio measurements. It necessary to say that 
Table 1. Observations used for fitting EPM2014 and estimating its parameters.

\begin{tabular}{|c|c|c|c|c|}
\hline & \multicolumn{2}{|c|}{ Radio } & Optical \\
\hline Planet & $\begin{array}{c}\text { Interval of } \\
\text { observations }\end{array}$ & $\begin{array}{c}\text { Number of } \\
\text { norm. points }\end{array}$ & $\begin{array}{c}\text { Interval of } \\
\text { observations }\end{array}$ & $\begin{array}{c}\text { Number of } \\
\text { observations }\end{array}$ \\
\hline Mercury & $1964-2009$ & 757 & - & - \\
Venus & $1961-2013$ & 3799 & - & - \\
Mars & $1965-2014$ & 47755 & - & - \\
Jupiter+4 sat. & $1973-1997$ & 51 & $1914-2013$ & 14866 \\
Saturn+7 sat. & $1979-2014$ & 188 & $1913-2013$ & 16455 \\
Uranus+4 sat. & 1986 & 3 & $1914-2013$ & 12550 \\
Neptune+1 sat. & 1989 & 3 & $1913-2013$ & 12404 \\
Pluto & - & - & $1914-2013$ & 16674 \\
\hline In total & $1961-2014$ & 52556 & $1913-2013$ & 72049 \\
\hline
\end{tabular}

whereas in Table 1 there are the number of optical observations, there are the number of normal points for radar observations, as tracking spacecraft data are correlated for each pass, so only one independent range point is used for the pass.

Observations are classical and modern optical observations of the outer planets and their satellites (since 1913), ranging to planets, the martian landers and spacecraft, including the data of Mariner-9, Viking-1,2, Pathfinder, MGS, Odyssey, MRO, Venus Express and Mars Express (1961-2014). The most part of the new data was obtained through the courtesy of Dr. W. Folkner (JPL) and Dr. A. Fienga (IMCCE).

The accuracy of trajectory measurements improved to several meters for today's martian spacecraft data and to about $20 \mathrm{~m}$ for Cassini (relative error is $10^{-12} \div 10^{-11}$ ). The modern optical data are CCD observations, and the accuracy of the best CCD data reaches 0. . 05 .

The progress in the accuracy of planet ephemerides depends on several factors: the adequacy of dynamic model of planetary motion to the real motion of planets, the quantity and quality of observational data. The new version of the ephemerides EPM2014 is being developed on the base of the EPM2011 ephemerides, described in detail in the paper (Pitjeva 2013). The main improvements of EPM2014 are:

- the number of observations has been increased - more than 800000 positional observations of planets and spacecraft (1913-2014) were used;

- data of asteroids and Trans-Neptunean Objects (TNO) have been updated and refined; in particular, we used the new mass value of Vesta obtained from Dawn spacecraft data orbiting Vesta, as well as data of double asteroids or asteroids having satellites;

- the usage of a massive two-dimensional asteroid annulus with dimension corresponding to observable data for modeling the total perturbation of the small asteroids, instead of a one-dimensional asteroid ring of the EPM2011.

\section{Estimations of individual mass values of the largest asteroids}

Estimates of masses of the largest asteroids can be found by analysis of high-accuracy radio data of Mars landers and orbiters using high-precision planetary ephemeris. The masses of Ceres, Pallas, Vesta were obtained by this method and accepted by the IAU as the best values (Luzum et al., 2011). The accurate values of the masses of the three the largest asteroids are very important, because these objects are more than half of the total mass of the main belt. The same method was used to estimate the masses of many other asteroids using ephemerides DE (Folkner et al., 2014), INPOP (Fienga et al., 2015), and EPM (Pitjeva \& Pitjev, 2014). 
Table 2. Individual estimations of asteroid masses obtained from radar data for EPM2014 in $10^{-12} M_{\odot}$.

\begin{tabular}{|lc|lc|}
\hline Asteroid & Mass & Asteroid & Mass \\
\hline (1) Ceres & $472.17 \pm 0.79$ & $(15)$ Eunomia & $14.45 \pm 0.55$ \\
(2) Pallas & $104.72 \pm 0.92$ & $(16)$ Psyche & $12.75 \pm 1.03$ \\
(3) Juno & $14.67 \pm 0.25$ & $(19)$ Fortuna & $4.36 \pm 0.13$ \\
(4) Vesta & $129.70 \pm 0.45$ & $(23)$ Thalia & $1.24 \pm 0.21$ \\
(4) Vesta-Dawn & $130.26846 \pm 0.00009$ & $(29)$ Amphitrite & $5.39 \pm 0.50$ \\
(6) Hebe & $4.05 \pm 0.46$ & $(41)$ Daphne & $4.17 \pm 0.44$ \\
(7) Iris & $6.54 \pm 0.30$ & $(52)$ Europa & $9.06 \pm 1.32$ \\
(8) Flora & $2.05 \pm 0.18$ & $(324)$ Bamberga & $5.10 \pm 0.14$ \\
(9) Metis & $1.63 \pm 0.25$ & $(511)$ Davida & $6.11 \pm 1.74$ \\
(10) Hygiea & $41.61 \pm 1.34$ & $(532)$ Herculina & $7.07 \pm 0.62$ \\
(14) Irene & $3.61 \pm 0.28$ & $(704)$ Interamnia & $12.22 \pm 0.96$ \\
\hline
\end{tabular}

The best mass values are available for a number of asteroids, to which spacecraft approached. The Mass of Vesta was estimated with the best accuracy from the Dawn spacecraft data (Konopliv et al., 2014). Masses of some asteroids, which are known as double or having satellites, were obtained with the sufficiently good accuracy. In addition, estimates of asteroid masses can be obtained from their close approaches, but in this case, as a rule, optical observations are used and the accuracy of mass estimates is worse. Individual estimates of the masses of asteroids can be obtained from their known diameters and the mean estimated density for the three taxonomic types $(\mathrm{C}, \mathrm{S}, \mathrm{M})$ while fitting ephemerides to observations. In this case their mass uncertainties are larger, reaching for the worst the cases $\sigma \sim 0.7 \cdot 10^{-12} M_{\odot}$.

For EPM, the 35 asteroid masses were calculated from their perturbations of Mars and the Earth (Tab. 2), from data of spacecraft, or from observations of asteroid satellites. Masses for 266 asteroids were derived from the estimated mean densities $\left(\rho_{C}=(1.12 \pm\right.$ $\left.0.04) \mathrm{g} / \mathrm{cm}^{3}, \rho_{S}=(1.78 \pm 0.09) \mathrm{g} / \mathrm{cm}^{3}, \rho_{M}=(3.4 \pm 0.41) \mathrm{g} / \mathrm{cm}^{3}\right)$ and the diameters known from infrared data. The total number of asteroid masses estimated individually is 301. To determine the masses of these asteroids, the radar observational material from Table 1 was used. The asteroids of the main belt affect significantly the motion of Mars. The correct analysis of this motion is possible due to the accumulated array of highaccuracy observations of spacecraft have been on the surface or have orbited around Mars (Viking, Pathfinder, MGS, Odyssey, MRO, MEX). Table 2 shows the improved estimates of the masses for 21 asteroids received while fitting the EPM2014 ephemerides to observations. The values of masses and densities, obtained by us, as well as diameters of asteroids were compared with the values from the paper by Carry (2012).

The total mass of the 301 asteroids estimated individually is $M_{301}=(11.186 \pm$ 0.095) $10^{-10} M_{\odot}$ including the masses of the 35 asteroids determined directly from their perturbations upon other bodies: $M_{35}=(8.271 \pm 0.036) \cdot 10^{-10} M_{S u n}$, and the total mass of the 266 asteroids estimated by their diameters and densities: $M_{266}=(2.915 \pm 0.088)$. $10^{-10} M_{\text {Sun }}$. All estimates of masses are given in this work with uncertainty equal $3 \sigma$ of the formal standard error of the least squares method.

\section{Modelling total attraction of small asteroids of the main belt}

The total mass of small main-belt asteroids was also evaluated by dynamical method. Although the mass of the main asteroid belt mostly belongs to large asteroids, for which "personal " mass estimations are carried out, modern high-precision ephemeris need to take into account the rest of the small bodies in the asteroid belt properly, adding up 
Table 3. The effect of a one-dimensional ring and two-dimensional asteroid annulus of the same mass $\left(10^{-10} M_{\odot}\right)$ on the additional perihelion advances, in milli-arcseconds for 10 revolutions

\begin{tabular}{|l|c|c|}
\hline Planet & $\begin{array}{c}\text { One-dimensional ring } \\
R=2.67 \mathrm{au}\end{array}$ & $R_{1}=2.06, R_{2}=3.27 \mathrm{au}$ \\
\hline Mercury & 0.003003110 & 0.003214501 \\
Venus & 0.021951638 & 0.025042493 \\
Earth & 0.067852470 & 0.075169552 \\
Mars & 0.383162773 & 0.497285998 \\
Jupiter & 0.460329887 & 0.539980540 \\
Saturn & 0.088503040 & 0.094955654 \\
\hline
\end{tabular}

to about $10 \%$ of the total mass. First of all, it affects the motion of Mars, whose orbit passes relatively close to the inner radius of the main belt.

Currently most of the existing asteroids larger than a few tens of kilometers are probably known, since they are sufficiently bright, even if they have the lowest albedo and are located on the outer edge of the belt (Zappala, Cellino 1996). However, this does not mean that one can obtain their accurate masses. There is a very large number of bodies with smaller sizes, including small asteroids and their fragments in the main belt. The destruction of asteroids occurs mainly in collisions or decay as a result of rotation, if an asteroid is a fairly loose conglomerate. Both processes produce new smaller asteroids (Bottke et al. 2005, Jacobson et al. 2014). In addition, the belt includes a dust component (Love \& Brownlee 1992; Reach 1992). To account for the impact of the numerous, mostly unseen bodies in the asteroid belt Krasinsky (Krasinsky et al. 2002) firstly proposed to model their combined gravitational influence by a uniform circular ring with parameters (the radius and mass) which can be determined by observations. This approach was implemented for ephemeris EPM2004 (Pitjeva 2005) and then in the following ephemeris: EPM2008 (Pitjeva 2010), EPM2011 (Pitjeva 2013). Because the asteroid belt is actually in a fairly wide circular band (the width is more than $1.2 \mathrm{au}$ ) and having the large array of precision observations, the model of the influence of small asteroids can be improved substantially by the transition from the usage of a one-dimensional homogeneous ring to the usage of two-dimensional annulus. The majority of small bodies is between 2.06 and $3.27 \mathrm{au}$, which corresponds to the resonant value of the distance of $4: 1$ and $2: 1$; beyond which the number of asteroids drops sharply.

Calculations show (Table 3) that the effect of a two-dimensional annulus differs notably from the effect of a one-dimensional ring of the same mass.

Considering that the asteroid belt occupies a broad annular area (the radial width is more than 180 million $\mathrm{km}$ ), modeling its gravitational effect by the two-dimensional homogeneous annulus makes it possible to take into account more properly the influence of numerous small asteroids.

Finding the gravitational potential and its derivatives for a two-dimensional uniform annulus leads to an expression involving complete elliptic integrals of the 1st, 2nd and 3rd kind (see, e.g., Duboshin, 1961). If we denote by $r=\sqrt{x^{2}+y^{2}+z^{2}}$ and $R=$ $\sqrt{x^{2}+y^{2}}$ corresponding distance, the expression for the potential of a homogeneous two-dimensional annulus $\left(R_{1}, R_{2}\right)$ with a mass $m$, is

$$
\begin{gathered}
U(R, z)=\frac{2 G m}{\pi\left(R_{2}{ }^{2}-R_{1}{ }^{2}\right)}\left[\sqrt{z^{2}+\left(R+R_{2}\right)^{2}} \cdot E\left(\chi_{2}\right)-\sqrt{z^{2}+\left(R+R_{1}\right)^{2}} \cdot E\left(\chi_{1}\right)\right. \\
-\frac{r^{2}-R_{2}{ }^{2}}{\sqrt{z^{2}+\left(R+R_{2}\right)^{2}}} K\left(\chi_{2}\right)+\frac{r^{2}-R_{1}{ }^{2}}{\sqrt{z^{2}+\left(R+R_{1}\right)^{2}}} K\left(\chi_{1}\right)
\end{gathered}
$$




$$
\begin{aligned}
& +\frac{\left(r-R_{2}\right)(r-R)}{\sqrt{z^{2}+\left(R+R_{2}\right)^{2}}} \Pi\left(\chi_{2},-n\right)-\frac{\left(r-R_{1}\right)(r-R)}{\sqrt{z^{2}+\left(R+R_{1}\right)^{2}}} \Pi\left(\chi_{1},-n\right) \\
& \left.+\frac{\left(r+R_{2}\right)(r+R)}{\sqrt{z^{2}+\left(R+R_{2}\right)^{2}}} \Pi\left(\chi_{2}, n^{\prime}\right)-\frac{\left(r+R_{1}\right)(r+R)}{\sqrt{z^{2}+\left(R+R_{1}\right)^{2}}} \Pi\left(\chi_{1}, n^{\prime}\right)\right],
\end{aligned}
$$

where

$$
\chi_{1}{ }^{2}=\frac{4 R_{1} R}{\sqrt{z^{2}+\left(R+R_{1}\right)^{2}}}, \quad \chi_{2}{ }^{2}=\frac{4 R_{2} R}{\sqrt{z^{2}+\left(R+R_{2}\right)^{2}}}, \quad n=\frac{2 R}{r+R}, n^{\prime}=\frac{2 R}{r-R} .
$$

Here $K(\chi), E(\chi)$ and $\Pi(\chi, b)$ are complete elliptic integrals of the 1st, 2nd and the third kind, respectively.

Terms of the equation (1) contain elliptic integrals $\Pi(\chi, b)$ tending to zero as $z \rightarrow 0$. In our case, the contribution of these terms is small even for Mars and Jupiter, the closest planets to the modeled belt, and it is by several orders of magnitude less than the contribution of terms with integrals of $K(\chi)$ and $E(\chi)$. The calculation of the remaining terms in the expression (1) and their derivatives can be simplified using the Landen conversion (Abramowitz \& Stegun 1965). In this case finding the complete elliptic integrals can be replaced by calculating the values of hypergeometric functions of several parameters. The expressions for the potential and the acceleration of the two-dimensional annulus $\left(R_{1}, R_{2}\right)$ are most simple when the attracted point and the attracting ring lie in same plane. The expressions for the accelerations in the plane $x, y$, for the points having radial distance of $r=\sqrt{x^{2}+y^{2}}$ inside or outside the annulus $\left(R_{1}, R_{2}\right)$ of mass $m$ are given in the work (Pitjeva \& Pitjev 2014).

For obtaining a more reliable estimate of the mass of the modelled annulus and its uncertainty, the three test versions of annulus dimensions are considered: $R_{1}=1.90$ au, $R_{2}=3.27 \mathrm{au} ; R_{1}=2.06 \mathrm{au}, R_{2}=3.50 \mathrm{au}$; and $R_{1}=1.90 \mathrm{au}, R_{2}=3.50$ au in addition to the basic annulus with dimensions $R_{1}=2.06 \mathrm{au}, R_{2}=3.27 \mathrm{au}$. For all these versions, the small differences of their masses (about 10\%) were found.

The following estimation of the mass of the basic two-dimensional annulus has been obtained:

$$
M_{\text {anullus }}=(1.059 \pm 0.161) \cdot 10^{-10} M_{\odot} .
$$

\section{The primary results and conclusion}

The obtained estimation of the total mass of a small asteroids, fragments, meteoroids and dust is about $9.5 \%$ of the mass of the 301 largest asteroids of the main belt. The total mass of the main belt including the 301 largest asteroids and the asteroid annulus is

$$
M_{\text {belt }}=(12.25 \pm 0.19) \cdot 10^{-10} M_{\odot} \quad\left(\approx 2.5 M_{\text {Ceres }}\right) .
$$

The comparison of the current and the previous estimates of the total mass of the main asteroid belt obtained by different authors are shown in Table 4 . It can be seen from Table 4 that the estimated value $M_{b e l t}$ became smaller.

The transition to the modeling of the total gravitational influence of small asteroids by the two-dimensional annulus with dimensions corresponding to the observed width of the main belt leads to a better representation of observations and decrease of uncertainty of the total mass of the asteroid belt. The standard error of the mass of the annulus of 
Table 4. Previous and recent estimates of the total mass of the main asteroid belt

\begin{tabular}{|c|c|c|}
\hline Year of public. & Authors & Mass (in $\left.M_{\odot}\right)$ \\
\hline 1990 & McBride \& Hughes & $\sim 55 \cdot 10^{-10}$ \\
2002 & Krasinsky et al. & $(18 \pm 2) \cdot 10^{-10}$ \\
2002 & Petit et al. & $15 \cdot 10^{-10}$ \\
2005 & Pitjeva & $(15 \pm 1) \cdot 10^{-10}$ \\
2012 & Vinogradova & $13.5 \cdot 10^{-10}$ \\
2013 & Kuchynka \& Folkner & $(13.6 \pm 0.2) \cdot 10^{-10}$ \\
2013 & Pitjeva & $(12.3 \pm 1.2) \cdot 10^{-10}$ \\
2014 & Pitjeva \& Pitjev & $(12.2 \pm 0.2) \cdot 10^{-10}$ \\
\hline
\end{tabular}

small asteroids and the total mass of the belt has decreased by six times compared with previous estimates using the one-dimensional ring (Pitjeva 2005, 2010, 2013).

This work was supported by the research grant 6.37.341.2015. of Saint Petersburg State University and the RAS Presidium Program 9 of fundamental researches "Experimental and theoretical researches of objects in the solar system and planet systems of stars".

\section{References}

Abramowitz, M. \& Stegun, I. A. 1965, Handbook of mathematical functions with formulas, graphs and mathematical tables, Dover Books on Advanced Mathematics, New York: Dover

Bottke, W. F., Durda, D. D., Nesvorny D., Jedicke, R., Morbidelli, A., Vokrouhlicky, D., \& Levison, H. F. 2005, Icarus, 179, 63

Carry, B. 2012, Planetary \& Space Science, 73, 98

Duboshin, G. N. 1961, Theory of attraction, Moscow: GIFML (in Russian)

Fienga, A., Manche, H., Laskar, J., Gastineau, M., \& Verma, A. 2015, Eprint arXiv:1405.0484, $29 \mathrm{p}$.

Jacobson, S. A., Marzari, F., Rossi, A., Scheeres, D. J. \& Davis, D. R. 2014, MRAS (Letters), 439, L95

Folkner, W. M., Williams, J. G., Boggs D. H., Park, R. S., \& Kuchynka, P. 2014, Interplanetary Network Progress Report, 42-196, 81 p.

Konopliv, A. S., Asmar, S. W., Park, R. S., et al. 2014, Icarus, 240, 103

Krasinsky, G. A., Pitjeva, E. V., Vasilyev, M. V., \& Yagudina, E. I. . 2002, Icarus, 158, 98

Kuchynka, P. \& Folkner, W. M. 2013, Icarus, 222, 243

Love, S. G. \& Brownlee, D. E. 1992, Astronomical Journal, 104, 2236

Luzum, B., Capitaine, N., Fienga, A., Folkner, W., Fukushima, T., Hilton, J., Hohenkerk, C., Krasinsky, G., Petit, G., Pitjeva, E., Soffel, M., \& Wallace, P. 2011, Celest. Mech. \& Dyn. Astr., 110, 293

McBride N. \& Hughes D. W. 1990, MNRAS, 244, 513

Petit, J.-M., Morbidelli, A., \& Chambers, J. 2001. Icarus, 153, 338

Pitjeva, E. V. 2005, Solar System Research, 39, 176

Pitjeva, E. V. 2010, in: S. A. Klioner, P. K. Seidelmann \& M. H. Soffel (eds.), Relativity in Fundamental Astronomy: Dynamics, Reference Frames, and Data Analysis, Proc. IAU Symposium No. 261 (Cambridge: Univ. Press), p. 170

Pitjeva, E. V. 2013, Solar System Research, 47, 386

Pitjeva, E. V. \& Pitjev, N. P. 2014, Celest. Mech. Dyn. Astr., 119, 237

Reach, W. T. 1992, Astrophysical Journal, 392, 289

Tedesco, E. F. \& Desert, F.-X. 2002, Astronomical Journal, 123, 2070

Tedesco, E. F. \& Cellino, A., Zappala V. 2005, Astronomical Journal, 129, 2869

Vinogradova T. 2012, Trudy Inst. Prikl. Astr. RAN, 26, 110

Zappala, V. \& Cellino, A. 1996, in T. W. Rettig \& J. M. Hahn (eds.) Completing the Inventory of the Solar System, Proc. Astonomical Society of the Pacific Conference No. 107, p. 29 